\title{
CREENCIAS IRRACIONALES Y ASERTIVIDAD DOCENTE CON ACTOS DE VIOLENCIA EN ESCOLARES
}

\author{
IRRATIONAL BELIEFS AND TEACHING ASSERTIVENESS WITH VIOLENCE IN SCHOOLS
}

\author{
Oswaldo Orellana M*., Lupe García A., Elisa Yanac R., Jorge Rivera M., Julio Alvites R., \\ Daphne Orellana G., José Perea R., Genaro Araujo S. Cristina Mendoza S. \\ UNMSM, LIMA - PERÚ
}

(RECIBIDO 15/03/13 AcEPTADo 16/06/13)

\begin{abstract}
RESUMEN
La meta de buscar indicadores cognitivos, afectivos y sociales en las buenas prácticas docentes, motivan la presente investigación de carácter descriptiva y correlacional.

Las tres variables seleccionadas han sido estudiadas con instrumentos que evalúan las creencias irracionales, la asertividad y el criterio de juicio hacia los actos de violencia escolar. Los dos primeros con formatos que gozan de validez y confiabilidad realizada por los propios autores e innumerables investigaciones; y el tercero una construcción específica para la presente investigación; los mismos que han sido sometidos al análisis de consistencia estadística, así como del contenido y extensión conceptual de los sistemas teóricos que lo sustentan.
\end{abstract}

El problema de investigación respondió a la siguiente interrogante: ¿Qué tipo de relación existe entre las creencias irracionales y la asertividad con los actos de violencia en escolares?

El marco teórico conceptual proviene de la teoría de la reestructuración cognitiva, propuesto por Albert Ellis que sustenta la existencia de distorsiones cognitivas debido a la presencia de creencias irracionales en la Interpretación de los actos humanos. Del mismo modo, la segunda variable, la asertividad proviene de la teoría de las habilidades sociales, que aportan con modelos de comunicación entre las personas, particularmente de la comunicación interpersonal del profesor y del alumno; estableciendo un continuo de narraciones que configuran situaciones típicas de violencia escolar.

De los hallazgos obtenidos podemos precisar que las correlaciones de las creencias irracionales (10) con las otras variables involucradas, actos de violencia y asertividad, son múltiples y no uniformes, pero con un común denominador, son directos cuando la creencia irracional es elevada con el juicio adverso al manejo del conflicto $\mathrm{y} / \mathrm{o}$ a la actitud punitiva del profesor y son opuestos cuando la creencia irracional es de menor intensidad y hay un trato humano y formativo de parte del profesor, es decir, en este último caso, la actitud o la respuesta formativa del profesor se encuentra asociada a la responsabilidad de criterio que el profesor despliega en su misión orientadora y altruista con los estudiantes. Así se puede observar en los indicadores cuantitativos de la correlación, expuesta en cuadros discretamente elaborados.

Palabras clave: asertividad, creencias irracionales, profesor tutor, violencia escolar 


\begin{abstract}
The goal of search indicators cognitive, affective and social good teaching practices, research motivate this descriptive and correlational in nature.

The three selected variables have been studied with instruments that assess irrational beliefs, assertiveness and the judgment criterion was the school violence. The first two formats that validity and reliability enjoyed by the authors and countless investigations, and the third a specific construction for this research, the same that have been subjected to analysis of statistical consistency, and the content and conceptual extension theoretical systems that sustain

The research problem answered the following question: What kind of relationship exists between irrational beliefs and assertiveness with violence in school?

The conceptual framework derived from the theory of cognitive restructuring, proposed by Albert Ellis which supports the existence of cognitive distortions due to the presence of irrational beliefs in the interpretation of human actions. Similarly, the second variable, assertiveness comes from the theory of social skills, which provide models of communication between people, particularly interpersonal communication teacher and student, establishing a continuum of narratives that shape typical situations school violence.

From the findings we point out that correlations of irrational beliefs (10) with the other variables involved, violence and assertiveness, are multiple and non-uniform, but with a common denominator, are direct when irrational belief is high with the judgment adverse to conflict management and/or punitive attitude of the teacher and are opposite when the irrational belief is no less intense and humane treatment and training of the teacher, that is, in the latter case, the attitude or response teacher training is associated with the responsibility of the teacher displays criteria in its altruistic mission and guiding students. This can be seen in the quantitative indicators of the correlation, presented in tables discreetly made.
\end{abstract}

Keywords: assertiveness, irrational beliefs, professor tutor, school violence

\title{
INTRODUCCIÓN
}

En las líneas de investigación del Instituto de Investigaciones Psicológicas de la Universidad Nacional Mayor de San Marcos, una de las primeras prioridades se encuentra en investigar la violencia en todas su manifestaciones, con el objetivo de prevenirla y contribuir a la construcción de una cultura de paz.

Declaraciones nacionales e internacionales, tales como las metas para el siglo XXI, de los Ministros de Educación de los distintos países, firmada en el Salvador (19 de mayo de 2008); en el Perú las intervenciones del Consejo Nacional de Educación el 27 de octubre 2010 que dio a conocer al país las "propuestas de políticas de educación del CNE banderas para el periodo 2011-2016, coinciden en que el profesor debe de estar preparado para orientar de manera integral a las nuevas generaciones, en beneficio de la instauración de programas de convivencia, ejercicio democrático y ciudadanía.

Así mismo investigadores sobre el tema pedagógico sostienen que "los procesos de orden, de disciplina o de control se ha de apoyar en una organización escolar que favorezca su realidad y que se refiere en un "clima de centro y de aula positivo, y en donde los profesores deben de tomar la iniciativa y el protagonismo"(Fernández 
2005); además contextualizadas con la ley 29719, de "convivencia sin violencia en las instituciones educativas", conocida en nuestro medio como "Ley Antibullying (2011), que abordan algunas expresiones de violencia escolar.

La presente investigación se centraliza en el estudio cognitivo de las creencias irracionales, como una manifestación de cierta perturbación en el pensamiento para opinar valorativamente ciertas situaciones específicas de la vida humana, como es el caso de los actos de violencia. A estos criterios agregamos la asertividad como una capacidad para expresar lo que una persona piensa y siente, sobre una situación específica, de valoración positiva o de la valoración negativa, pero que es necesario expresarla en el momento oportuno, sin diferirla o reaccionando impulsivamente in situ, que implica también el resguardo de los derechos formativos de los estudiantes.

En dicha línea la presente investigación se plantea los siguientes objetivos: (1) Estudiar el tipo de relación que existe entre las creencias irracionales y la asertividad docente con los actos de violencia en escolares, (2) Identificar las creencias irracionales dominantes relacionadas con la asertividad, (3) Describir los grupos de creencias irracionales relacionadas con las conductas asertivas.

Las metas consecuentes se plantearon en los siguientes términos: (1) Evaluar una muestra de profesores de educación secundaria de Lima Metropolitana, que laboran en el área urbana y áreas de menor desarrollo, (2) Realizar un análisis de consistencia a los ítems de tres instrumentos relacionados con el área cognitiva, de habilidades sociales y juicio a conductas de violencia, (3) Establecer la diferencia de criterios por género de profesores hacía los actos de violencia.

\section{MARCO TEÓRICO}

Albert Ellis (1992) aborda el pensamiento como creencias, que son ideas, autoafirmaciones o imágenes determinantes primarias de las emociones, distinguiendo dos manifestaciones cognitivamente diferenciadas, los pensamientos racionales y las creencias irracionales. Los primeros, basados en una racionalidad filosófica para entender el mundo de una manera armoniosa, que distingue la bondad y la maldad en la mente y en la conducta humana, y que favorece a los individuos para alcanzar determinadas metas y objetivos, a través de regulaciones y/o autorregulaciones, con soportes socioemocionales, adecuadamente controlados. Y a la inversa encontramos las creencias irracionales, que constituyen distorciones de la realidad y configuran una personalidad con demandas excesivas, a través de un proceso de etiquetar a las personas, y que actúan como bloqueadores y por lo tanto, obstáculos para el logro adecuado de las interacciones interpersonales, y que en su expresión más inmediata afectan la convivencia y la salud mental normal.

Para enterder mejor la mente humana, Ellis (2000) plantea la técnica de reestructuración cognitiva, con el objetivo de intervenir en las personas que "cultivan" las creencias irracionales, como una expresión de distorsiones cognitivas, que involucran las emociones y conductas resultantes directas de la gestión mental del pensamiento. 
Consideramos que en el campo formativo, la mente de los profesores de la educación básica no son ajenos a la explicación de Ellis; más aún cuando gran parte de su actividad está orientada a juzgar conductas de los estudiantes y orientar su actuación; es decir que también asumen el riesgo de manifestaciones de distorsiones cognitivas, las cuales hay que estudiar en su real dimensión.

Las diez creencias irracionales involucradas en la investigación (Ellis), son las siguientes : (1) Para mi existe una necesidad absoluta de cariño y aprobación de mis semejantes, familia y amigos; (2) Debo ser altamente competente y casi perfecto en todo lo que emprendo; (3) Ciertas personas son malas y viles y perversas y deberían ser castigadas; (4) Es horrible cuando las personas y las cosas no son como uno quisieran que fueren; (5) Los acontecimientos externos son la causa de la mayoría de las desgracias de la humanidad, la gente simplemente reacciona según como los acontecimientos inciden sobre sus emociones; (6) Hay que sentir miedo o ansiedad ante cualquier cosa desconocida, incierta o potencialmente peligrosa; (7) Es más fácil evitar problemas y responsabilidades de la vida que hacerles frente; (8) Se necesita contar con algo o alguien más grande y más fuerte que uno mismo; (9) El pasado tiene que mucho que ver en la determinación del presente; (10) La felicidad aumenta con la inactividad, la pasividad y el ocio indefinido.

Sobre la violencia escolar tomamos como antecedente la investigación Estudio sociométrico de escolares que participan en actos de violencia (Orellana, Oswaldo y Orellana G., Daphne, 2010) realizado en un colegio público secundario de Lima Metropolitana, con la referencia de tener antecedentes de participar en violencia callejera a través de "pandillas escolares; según la investigación la incidencia se focalizó en diez secciones; las cuales sumaban 320 alumnos, de $3 .{ }^{\text {ro }}$ y $4 .{ }^{\text {to }} \mathrm{de}$ secundaria; de un total de 3,500 alumnos matriculados en los turnos de mañana y tarde, registrado en el departamento de Psicología, que mantenía un programa de prevención de la violencia en los niveles de primaria, secundaria y terciaria, es decir programa orientados a evitar el surgimiento de la violencia, aislar los factores de riesgo y el tratamiento especializado a nivel de casuística, respectivamente. Se utilizó como instrumento el test sociométrico (Moreno) aplicado a las aulas involucradas en actos de violencia, identificadas en una casuística que sistemáticamente se había establecido para la orientación pedagógica, individual y familiar. Los objetivos del Test Sociométrico han sido los siguientes: (1) Investigar la ubicación de cada uno de los miembros del aula, en función del grado de cohesión, logradas por las relaciones interpersonales establecidas; (2) Descubrir el papel del líder en la integración grupal, así como las motivaciones manifiestas de los miembros del aula; (3) Detectar los subgrupos generados "espontáneamente"; (4) Identificar a los alumnos rechazados, aislados y marginados. Es importante recordar que el test sociométrico, Identifica la cohesión del grupo y sus líderes; Identifica también a los olvidados, marginados y rechazados. El criterio de rechazo a está establecido porque afectan a la estabilidad del grupo. Las razones más frecuentes son por su agresividad a compañeros y el clima de hostilidad que generan. Aquí algunos testimonios sistematizados por el estudio de investigación "El (...) piensa que el más fuerte son los compañeros que se burlan de los demás, humillan a los más débiles y rompen los cuadernos y el mobiliario escolar, física- 
mente trasmiten una imagen de dominación y en su lenguaje agresivo, permanentemente están sometiendo al grupo". En los resultados del estudio encontramos que más del $50 \%$ se elige entre sí mismos... interpretando por lo tanto una ausencia de liderazgo positivo o estrella del aula, tal vez como una respuesta de defensa y de protección mutua. En cuanto al comportamiento sociométrico de los subgrupos disociales en cuanto al reconocimiento de "camarillas", se ubican , "estructuras con líderes definidos", tipificadas por las respuestas entre cero y $20 \%$, lo que caracteriza la existencia de "camarrillas" cohesionados para protegerse entre sí mismos, en contradicción a lo esperado, para grupos medianamente integrados (entre 25 a $49 \%$ ); en cuanto a los "líderes populares" se expresa la misma tendencia para tipificar al líder positivo, donde solamente se puede identificar a varios líderes populares, es decir que tienen tres votos; reiterando que no se pueda identificar a las estrellas, pues estos deberían de obtener más de ocho votos. El líder disocial, sociométricamente es el rechazado expresado en la elección por encima del $80 \%$ de los alumnos de un salón de clase, por ser identificado por el protagonismo en los actos de violencia, específicamente por desestabilizar el grupo escolar en formación, violar los derechos de sus compañeros y alterar las normas de convivencia, entre otros hallazgos, que tipifican relaciones de autorismo en los grupos que se forman en los actos de violencia escolar, preocupante por bloquear el clima formativo en el área de ciudadanía, de conductas prosociales y habilidades sociales.

Alberto Bandura (1988) en su teoría sobre el modelo de causalidad en el aprendizaje social sostiene que "la gente procesa y transforma las experiencias pasajeras en modelos internos a través de los símbolos, que sirven como guías para la acción futura. De la misma forma, las personas a través de los símbolos dan significado, forma y continuación de las experiencias que viven”, agregando “... si la gente sabe razonar lógicamente, hacen juicios erróneos cuando basan sus inferencias en una información inadecuada, o fracasan al considerar las consecuencias finales de elecciones diferentes. Más aun, las personas a menudo interpretan mal los hechos, de forma que dan lugar a falsas concepciones sobre ellos mismos y el mundo que los rodea. Cuando actúan a partir de sus concepciones erróneas que parecen subjetivamente racionales dada su base errada, tales personas se comportan, en opinión de los demás, de una forma poco razonable, sino totalmente alocada.

Sobre el concepto de violencia, es necesario distinguirlo de la agresividad. En el primer caso la violencia es un fenómeno interpersonal, de dominación de un sujeto sobre otro, de un grupo sobre otro, de una clase social sobre otra. Afecta a dos protagonistas, quién la ejerce y quien la padece, mientras que la agresividad según K. Lorenz, es un "componente inevitable de la vida", está relacionada con la tendencia sadomasoquista cuya finalidad es destruir o humillar al otro, y también a sí mismo (autoagresividad, desprendiéndose que la violencia tiene una fuerte connotación social y la agresividad, una connotación individual. Una explicación de la violencia, tiene necesariamente que existir una relación de dependencia entre dos personas: "Es una violencia que se ejerce sobre los individuos con su propia complicidad. Existe una diversidad de formas en las que se ejerce la violencia simbólica... los esquemas mentales y culturales que funcionan como una matriz simbólica de la práctica social, se convierten en un verdadero fundamento de la teoría del poder". 
Específicamente sobre el comportamiento juvenil, tomamos el concepto generacional para analizar sus manifestaciones de una manera global y transcendente y citamos a Janser Nerina (1977) para establecer que una generación es "un determinado conjunto social de perspectivas, desarrollado por los coetáneos que comparten las mismas circunstancias, o la forma social en que existen las perspectivas desarrolladas por coetáneos que comparten las mismas circunstancias". Concepto sociológico que lo enriquecemos con la psicología evolutiva sobre la adquisición de la autonomía, opuesto a la heteronomía, y que genera tensión con "las otras generaciones", o mejor dicho, la generación adulta, en nuestro caso, de los profesores; motivando en la mayoría de las veces conflictos de influencia y autoridad, en el sentido de juicio de las conductas. El tema se hace específicamente más complejo y requiere de cierta racionalidad para entenderlo integralmente.

La premisa que empleamos en las relaciones generacionales de profesor-estudiante, es que los profesores de secundaria asumen una responsabilidad formativa, y como tal deben desarrollar habilidades sociales de buena comunicación. Investigadores como Caballo (1991), Castanyer, 1996), Cuell (2000) Haddou (2001), Lazarus (1990), Michelson (1987), Rogers (1990), han investigado y creado programas específicos para mejorar la comunicación interpersonal, privilegiando a la asertividad como la capacidad para expresar lo que una persona piensa y siente en el momento oportuno, sin agredir a los demás y resguardando su integridad.

Ubicamos así dos variables psicológicas que las relacionamos para elaborar un perfil de orientación escolar y el trabajo tutorial de los profesores de Educación básica; a las cuales le agregamos un escenario de actuación, a nivel de opinión o criterio narrativo; esto es, a partir de escenas de violencia escolar en situaciones típicas de agresión, conflicto, dominación o efectos de maltrato en los escolares. Esto último también investigado por autores como Díaz Aguado (2006), La Madrid (2007). Nizama (2003) y por entidades que abordan el bienestar, de los sectores de Educación y Salud, como es el Plan Nacional de Salud Mental (2005).

Sobre el papel formativo de los profesores a los jóvenes, hay que tener en cuenta la línea de la formación ética, sobre todo aquellos criterios y comportamientos relacionados con la violencia en sus distintas manifestaciones en los ambientes escolares. Al respecto hay que tener en cuenta la socialización de las generaciones en la violencia como espectáculo, promovidas por los medios de comunicación, fácilmente identificados en los videos y en el cine comercial. Más atrás ha quedado la violencia política en la práctica del terrorismo (1980 hacia adelante), que las generaciones actuales no la han percibido vivencialmente, sino que ha quedado en la memoria histórica, trasmitidas oralmente por sus padres y profesores

Es importante señalar que el Ministerio de Educación destaca tres conflictos éticos (2005), para ser abordados por los profesores en el contexto social; (1) La corrupción; (2) la discriminación; y (3) La violencia social.

La respuesta del profesor sobre el juicio de los actos de violencia, son respuestas de carácter éticos. De La teoría de Kolhberg se desprende que la labor docente debe enfocarse a facilitar a los estudiantes un punto de vista más amplio que incluya -además de las normas y reglamentos y la obediencia debida a ellos- los 
principios éticos universales que deberían sustentar dichas normas, que corresponden al nivel post-convencional. También es denominado por Kohlberg como nivel "de principios". En él hay un claro esfuerzo por definir valores y principios morales que tienen validez y aplicación con independencia de la autoridad de los grupos o personas que mantienen tales principios, e independientemente de la propia identificación de las personas, propios de la función formativa docente.

\section{EL PROBLEMA DE INVESTIGACIÓN}

¿Qué tipo de relación existe entre las creencias irracionales y la asertividad con los actos de violencia en escolares?

\section{MÉTODO}

El método empleado en la presente investigación es el correlacional y tiene como objetivo precisar las asociaciones establecidas en las variables involucradas.

\section{Diseño de investigación}

- Descriptivo y correlacional

\section{Muestreo}

- La muestra seleccionada es probabilística estratificada.

\section{Variables relacionadas}

- Creencias irracionales, evaluadas con el inventario de creencias irracionales de Albert Ellis.

- Asertividad docente, evaluadas con el cuestionario Lazarus y Kolkman.

- Opinión sobre actos de violencia escolar, evaluada con un instrumento narrativo construido con dicho objetivo.

\section{Variables controladas}

- Edad

- Género

- Ejercicio docente (inicial, primaria, secundaria, superior)

- Función de tutoría (tutor de aula, coordinador de tutoría)

- Institución educativa donde labora (pública, privada)

- Especialidad: ciencias básicas, ciencias sociales, matemáticas, humanidades, tecnología 
- Años de servicio

- Área de trabajo (urbana, urbana marginal, rural)

- Formación profesional universitaria, superior pedagógica, superior tecnológica, maestría, doctorado

- Estado civil : soltero, casado, conviviente, divorciado, otro

- Lugar de residencia : Lima, provincia

\section{Instrumentos de Investigación}

\section{- Inventario de creencias irracionales}

Nombre: Inventario de creencias irracionales

Autoría: Albert Ellis

Aplicación y duración: Individual y colectiva en un tiempo aproximado de 30 minutos

Objetivo: Evaluar creencias irracionales que son fuentes de conflicto en la valorización de hechos humanos

\section{- Test de asertividad}

Nombre: Cuestionario de asertividad

Autoría: Lazarus y Folkman

Aplicación y duración: Individual y colectiva en un tiempo aproximado de 10 minutos

Opinión sobre actos de violencia: Instrumento construido para la presente investigación y validado con el método de juicio de expertos.

Los resultados son expuestos de acuerdo a las variables planteadas, empezando por las variables de control, que tipifican la muestra de profesores investigados. Así tenemos que la prueba se aplicó a 256 profesores, ubicados en su lugar de trabajo, en las áreas seleccionadas con dicho objetivo, Lima urbana, Lima marginal y Lima sierra (Tabla N. ${ }^{\circ} 11$ ).

\section{RESULTADOS}

La población corresponde a profesores de Lima Metropolitana y de dicho universo tomamos una muestra de profesores que laboran en la educación pública (256 sujetos), de los cuales el $54.3 \%$ se ubica en el intervalo de 18 a 30 años de edad (Tabla N. ${ }^{\circ} 1$ ), distribuida en $2 / 3$ de la muestra perteneciente al género femenino y un cuarto al género masculino, en la siguiente relación $75.4 \%$ y $22.7 \%$, respectivamente (Tabla N. ${ }^{\circ}$ 2). 
Oswaldo Orellana M., Lupe Garcia A., Elisa Yanac R., Jorge Rivera M., Julio Alvites R. Daphne Orellana G et al.

Tabla N. ${ }^{\circ}$ 1. Edad de los profesores

\begin{tabular}{cc|cccc}
\hline & Frecuencia & Porcentaje & $\begin{array}{c}\text { Porcentaje } \\
\text { válido }\end{array}$ & $\begin{array}{c}\text { Porcentaje } \\
\text { acumulado }\end{array}$ \\
\hline \multirow{4}{*}{ De 18 a 25 } & 40 & 15.6 & 15.6 & 15.6 \\
De 26 a 30 & 99 & 38.7 & 38.7 & 54.3 \\
Vélidos 31a 35 & 49 & 19.1 & 19.1 & 73.4 \\
& De 36 a 40 & 38 & 14.8 & 14.8 & 88.3 \\
& De 41 a 45 & 14 & 5.5 & 5.5 & 93.8 \\
& De 46 a 50 & 7 & 2.7 & 2.7 & 96.5 \\
& Más de 50 & 1 & .4 & .4 & 96.9 \\
No especifica & 8 & 3.1 & 3.1 & 100.0 \\
\hline & 256 & 100.0 & 100.0 & \\
\hline
\end{tabular}

Tabla N. ${ }^{\circ}$ 2. Género de los profesores

\begin{tabular}{cc|cccc}
\hline & & Frecuencia & Porcentaje & $\begin{array}{c}\text { Porcentaje } \\
\text { válido }\end{array}$ & $\begin{array}{c}\text { Porcentaje } \\
\text { acumulado }\end{array}$ \\
\hline \multirow{4}{*}{ Válidos } & Masculino & 58 & 22.7 & 22.7 & 22.7 \\
& Femenino & 193 & 75.4 & 75.4 & 98.0 \\
& No especifica & 5 & 2.0 & 2.0 & 100.0 \\
& Total & 256 & 100.0 & 100.0 & \\
\hline
\end{tabular}

En cuanto al ejercicio de la práctica docente encontramos cierta uniformidad de la muestra, entre los profesores de educación básica de primaria y secundaria, 34.4\% y $38.3 \%$, y en menor medida los profesores dedicados a la educación inicial $21.5 \%$ (Tabla N. ${ }^{\circ} 3$ ). En cuanto a la función tutorial, destaca que el $77.0 \%$ desarrolla funciones de tutoría, hecho que particularmente nos interesa en la investigación, dado que aquí los profesores desarrollan criterios formativos y respuestas consecuentes al afrontamiento de los actos de violencia escolar (Tablas $\mathrm{N}^{0} 3$ y 4 )

Tabla N. ${ }^{0}$ 3. Ejercicio docente

\begin{tabular}{cc|cccc}
\hline & Frecuencia & Porcentaje & $\begin{array}{c}\text { Porcentaje } \\
\text { válido }\end{array}$ & $\begin{array}{c}\text { Porcentaje } \\
\text { acumulado }\end{array}$ \\
\hline \multirow{4}{*}{ Válidos } & Inicial & 55 & 21.5 & 21.5 & 21.5 \\
& Primaria & 88 & 34.4 & 34.4 & 55.9 \\
& Secundaria & 98 & 38.3 & 38.3 & 94.1 \\
& Superior & 3 & 1.2 & 1.2 & 95.3 \\
& Otros & 3 & 1.2 & 1.2 & 96.5 \\
& No especifica & 9 & 3.5 & 3.5 & \\
\hline
\end{tabular}


Tabla N. ${ }^{\circ}$ 4. Función de tutoría

\begin{tabular}{cc|c|c|c|c}
\hline & & Frecuencia & Porcentaje & $\begin{array}{c}\text { Porcentaje } \\
\text { válido }\end{array}$ & $\begin{array}{c}\text { Porcentaje } \\
\text { acumulado }\end{array}$ \\
\hline \multirow{4}{*}{ Válidos } & Aula & 197 & 77.0 & 77.0 & 77.0 \\
& No soy tutor & 34 & 13.3 & 13.3 & 90.2 \\
& No especifica & 25 & 9.8 & 9.8 & 100.0 \\
& Total & 256 & 100.0 & 100.0 & \\
\hline
\end{tabular}

Dos variables controladas se ha identificado en la investigación. Nos referimos a la Institución Educativa donde laboran los profesores y la especialidad de enseñanza bajo su responsabilidad. En el primer caso ubicamos que el $61.9 \%$ lo hacen en colegios de educación pública y $38.9 \%$ en educación privada (Tabla $\mathrm{N}^{0} 5$ ); que es más o menos misma tendencia de la educación en Lima Metropolitana. De otro lado tenemos la especialidad que ejercen en su función formativa y tenemos que el $55.1 \%$ lo hacen en la especialidad de ciencias básicas, en la que incluye la educación primaria, las matemáticas, ciencias biológicas y ciencias físicas principalmente y $39.7 \%$ lo hacen en ciencias sociales (Tabla N. ${ }^{\circ} 6$ ).

Tabla N. ${ }^{0}$ 5. Institución Educativa donde laboran los profesores

\begin{tabular}{c|cc}
\hline & Recuento & $\%$ del N. ${ }^{\circ}$ de la columna \\
\hline Institución educativa privada & 98 & $38.9 \%$ \\
Institución educativa pública & 156 & $61.9 \%$ \\
Total & 252 & $100.0 \%$ \\
\hline
\end{tabular}

Tabla N. ${ }^{0}$ 6. Especialidad del profesor

\begin{tabular}{l|cc}
\hline & Recuento & \% del N. ${ }^{\circ}$ de la columna \\
\hline Especialidad ciencias básicas & 129 & $55.1 \%$ \\
Especialidad ciencias sociales & 59 & $25.2 \%$ \\
Especialidad ciencias matemáticas & 27 & $11.5 \%$ \\
Especialidad comunicación & 34 & $14.5 \%$ \\
Total & 234 & $100.0 \%$ \\
\hline
\end{tabular}

Las variables de años de servicio y áreas de trabajo expresan que el $86.3 \%$ tienen menos de diez años de servicio (Tabla N. ${ }^{\circ} 7$ ) y que el $63.3 \%$ laboran en las áreas urbanas y urbanas marginales (Tabla N. ${ }^{\circ} 8$ ). 
Oswaldo Orellana M., Lupe Garcia A., Elisa Yanac R., Jorge Rivera M., Julio Alvites R. Daphne Orellana G et al.

Tabla N. ${ }^{\circ}$ 7. Años de servicio de los profesores

\begin{tabular}{cc|cccc}
\hline & Frecuencia & Porcentaje & $\begin{array}{c}\text { Porcentaje } \\
\text { válido }\end{array}$ & $\begin{array}{c}\text { Porcentaje } \\
\text { acumulado }\end{array}$ \\
\hline \multirow{4}{*}{ De 0 a 5 } & 149 & 58.2 & 58.2 & 58.2 \\
& De 6 a 10 & 72 & 28.1 & 28.1 & 86.3 \\
& De 11 a 15 & 22 & 8.6 & 8.6 & 94.9 \\
& De 16 a 20 & 7 & 2.7 & 2.7 & 97.7 \\
& De 21 a 25 & 4 & 1.6 & 1.6 & 99.2 \\
& No especifica & 2 & .8 & .8 & 100.0 \\
\hline
\end{tabular}

Tabla N. ${ }^{\circ}$ 8. Área de trabajo del profesor

\begin{tabular}{cc|cccc}
\hline & & Frecuencia & Porcentaje & $\begin{array}{c}\text { Porcentaje } \\
\text { válido }\end{array}$ & $\begin{array}{c}\text { Porcentaje } \\
\text { acumulado }\end{array}$ \\
\hline \multirow{4}{*}{ Válidos } & Urbana & 106 & 41.4 & 41.4 & 41.4 \\
& Urbana marginal & 56 & 21.9 & 21.9 & 63.3 \\
& Urbana rural & 82 & 32.0 & 32.0 & 95.3 \\
& No especifica & 12 & 4.7 & 4.7 & 100.0 \\
& Total & 256 & 100.0 & 100.0 & \\
\hline
\end{tabular}

Finalmente tres variables controladas importantes son la formación profesional del profesor, el Estado Civil y el lugar de residencia. El $70.6 \%$ afirma que su formación profesional de profesor la realizó en un Instituto Superior pedagógico y un $22.6 \%$ realizó sus estudios en la Universidad (Tabla N. ${ }^{\circ}$ 9). En cuanto al estado civil, $53.9 \%$ se encuentra en la categoría de soltero(a) (Tabla N. ${ }^{\circ} 10$ ). Sobre el lugar de trabajo, el $96.7 \%$ tiene su lugar de trabajo en Lima, ya sea en Lima ciudad, Lima marginal y/o Lima sierra (Tabla N. ${ }^{\circ} 11$ )

Tabla N. ${ }^{\circ}$ 9. Formación profesional del profesor

\begin{tabular}{c|cc}
\hline & Recuento & \% del N. $^{\circ}$ de la columna \\
\hline Universitaria en pedagogía & 50 & $22.6 \%$ \\
Superior pedagógico & 156 & $70.6 \%$ \\
Superior tecnológico & 14 & $6.3 \%$ \\
Maestría & 9 & $4.1 \%$ \\
Doctorado & 0 & $.0 \%$ \\
Otra especialidad & 5 & $2.3 \%$ \\
Total & 221 & $100.0 \%$ \\
\hline
\end{tabular}


Tabla N. ${ }^{0}$ 10. Estado civil del profesor(a)

\begin{tabular}{cc|cccc}
\hline & Frecuencia & Porcentaje & $\begin{array}{c}\text { Porcentaje } \\
\text { válido }\end{array}$ & $\begin{array}{c}\text { Porcentaje } \\
\text { acumulado }\end{array}$ \\
\hline \multirow{4}{*}{ Válidos } & Soltero & 138 & 53.9 & 53.9 & 53.9 \\
& Casado & 78 & 30.5 & 30.5 & 84.4 \\
& Conviviente & 29 & 11.3 & 11.3 & 95.7 \\
& Divorciado & 4 & 1.6 & 1.6 & 97.3 \\
& No especifica & 7 & 2.7 & 2.7 & 100.0 \\
& Total & 256 & 100.0 & 100.0 & \\
\hline
\end{tabular}

Tabla N. ${ }^{\circ}$ 11. Lugar de trabajo del Profesor (a)

\begin{tabular}{cc|cccc}
\hline & & Frecuencia & Porcentaje & $\begin{array}{c}\text { Porcentaje } \\
\text { válido }\end{array}$ & $\begin{array}{c}\text { Porcentaje } \\
\text { acumulado }\end{array}$ \\
\hline \multirow{4}{*}{ Válidos } & Lima Ciudad & 112 & 43.8 & 43.8 & 43.8 \\
& Lima Marginal & 78 & 30.5 & 30.5 & 74.2 \\
& Lima Sierra & 57 & 22.3 & 22.3 & 96.5 \\
& Selva & 5 & 2.0 & 2.0 & 98.4 \\
& No especifica & 4 & 1.6 & 1.6 & 100.0 \\
\hline
\end{tabular}

Los resultados detallados se encuentran expuestos en tablas de doble entrada en dos secciones anexadas en la presente investigación, no publicadas en el presente informe por razones de espacio, y que se exponen de la siguiente manera:

\section{Primera sección:}

1. Exposición de los resultados descriptivos de los 100 ítems de las creencias irracionales, en igual número de tablas (Tablas 12 a la 112).

2. Exposición de los resultados descriptivos de los 20 ítems de asertividad, en igual número de tablas (tablas de la 113 a 133).

3. Exposición de resultados descriptivos sobre la narrativa de opinión de actos de violencia escolar, en igual número de tablas (tablas 134 a 138).

\section{Segunda sección}

1. Tabla de correlaciones entre creencia irracional y actos de violencia, una tabla por cada creencia irracional, total 10 tablas (Tablas 139 a 1148).

Tabla de correlaciones entre asertividad y actos de violencia (Tabla 149).

Tabla de correlaciones entre creencia irracional y asertividad, total 10 tablas (Tablas 150 a 159). 
La exposición de los resultados sigue el marco lógico del establecimiento de las relaciones de las diez creencias irracionales con la asertividad y la narrativa de los actos de violencia escolar- Así tenemos que al observar las matrices de correlaciones entre los ítems de la variable Creencias Irracionales y los ítems de opinión frente a actos de violencia escolar en los estudiantes escolares, podemos describir lo siguiente:

\section{Creencia 1}

Solo es significativa una correlación entre el ítem 31 y el ítem 1 de actos de violencia, esta correlación es inversa (-0.140) interpretado quiere decir que los docentes mientras tengan hegemonía en su creencia irracional, mantienen el juicio represivo del profesor a los actos de violencia de los estudiantes, es decir que tiene dificultades para relativizar las conductas de los escolares-.

\section{Creencia 2}

Es significativa la correlación entre ítem 72 con el ítem $1(0.184)$ y el ítem 6 de actos de violencia (0.155) estas correlaciones son directas. Esto significa que la creencia Irracional 2, referida a ser "altamente competente y casi perfecto en todo lo que emprendo" es correcta la actitud represiva a los escolares que hacen actos de violencia (ítem 1 del relato), coherente con el ítem 6 del relato de inhibirse en la solidaridad con la ayuda que soplicita un alumno que es maltratado por otros compañeros.

\section{Creencia 3}

Resaltan las correlaciones significativas entre el ítem 83 con el ítem 1 (-0.128), con el ítem $2(0.105)$ y con el ítem $3(-0.140)$ de actos de violencia.; referida a la creencia irracional de la mladad de las personas y por ello merecen el castigo correspondiente.

\section{Creencia 4}

Es significativa la correlación entre el ítem 74 con el ítem 1 (-0.141), con el ítem 2 (0.188), referido a intervenir positivamente para afrontar la violencia de los escolares y con el ítem $3(-0.218)$, También resalta la correlación entre el ítem 84 con el ítem $2(0.164)$, con el ítem 4 (0.130), con el ítem $5(0.134)$ y con el ítem 6 (0.136) de indiferencia o falta de voluntad para ayudar a las víctimas de la violencia.

\section{Creencia 5}

Es significativa la correlación entre el ítem 65 con el ítem $1(-0.165)$, contrario a la represión de la violencia a escolares; y con el ítem $6(0.138)$ d. También resalta la correlación entre el ítem 75 con el ítem $2(0.160)$ y con el ítem $3(-0.134)$ de actos de violencia. La correlación 85 con el ítem $6(0.165)$ de actos de violencia. Por último el ítem 95 con el ítem $2(0.130)$, con el ítem $3(-0.193)$ y con el ítem $6(0.108)$ de actos de violencia, aunque no significativos, pero mantiene la confabulación de las cirsunstancias para explicar los actos de violencia. 
CREENCIAS IRRACIONALES Y ASERTIVIDAD DOCENTE CON ACTOS DE VIOLENCIA EN ESCOLARES

\section{Creencia 6}

Destaca la correlación significativa entre el ítem 36 con el ítem $1(0.194)$ y con el ítem $4(-0.127)$ de actos de violencia., abiertamente represivos a los estudiantes que realizan actos de violencia.

\section{Creencia 7}

Resalta la correlación significativa entre el ítem 7 con el ítem 1 (0.148) de actos de violencia; la correlación entre el ítem 17 con el ítem 1 (-0.195) de actos de violencia; la correlación entre el ítem 27 con el ítem 1 (0.166) de actos de violencia. La correlación entre el ítem 47 con el ítem $2(0.126)$ de actos de violencia. La correlación entre el ítem 77 con el ítem $1(-0.147)$, con el ítem $2(0.205)$ y con el ítem 3 (-0.161) de actos de violencia, que mantienen un común denominador con la evasión al afrontamiento de las tareas formativas de los estudiantes, es decir indiferencia para orientar la prevención de los actos violentos.

\section{Creencia 8}

Destaca la correlación significativa entre el ítem 48 con el ítem $1(-0.151)$, con el ítem $2(0.190)$ y con el ítem $3(-0.187)$ de actos de violencia. La correlación entre el ítem 58 con el ítem $2(0.186)$ y con el ítem $3(-0.164)$ de actos de violencia. La correlación entre el ítem 68 con el ítem 1 (-0.141), con el ítem $2(0.172)$ y con el ítem $3(-0.197)$ de actos de violencia. Las correlaciones positivas altas, demandan intervenciones superiores para atender los actos de violencia, y las correlaciones negativas, también altas, buscan la intervención represiva fuera del ámbito escolar.

\section{Creencia 9}

Destaca la correlación entre el ítem 19 con el ítem $1(0.135)$ y con el ítem 3 (0.159). La correlación entre el ítem 79 y el ítem 1 (0.102) de actos de violencia.; aunque no son altas, existe cierta fijación para explicar los actos de violencia por experiencias pasadas.

\section{Creencia 10}

Destaca la correlación significativa entre el ítem 20 y el ítem $3(0.127)$ de actos de violencia; la correlación significativa entre el ítem 50 y el ítem $1(0.132)$ de actos de violencia; la correlación entre el ítem 90 con el ítem $3(-0.135)$ de actos de violencia; por último la correlación significativa entre el ítem 100 y el ítem 1 (0.137) de actos de violencia. Correlaciones que tienen que ver con la inactividad para intervenir en los actos de violencia escolar.

\section{Asertividad y actos violentos}

Al analizar la matriz de correlaciones entre los ítems de la variable asertividad y los ítems de opinión frente a actos de violencia escolar, podemos describir lo siguiente:

Se puede observar 11 correlaciones significativas mayores a $20 \%$, entre ellas destacan las correlaciones significativas entre el ítem 1 de actos de violencia con 
los ítems 1 (0.201), 4 (-0.216), 7 (-0.202), 11 (0.278), 12 (-0.279), 16 (-0.269), $18(-0.255)$ y $20(-0.216)$ de asertividad.

\section{Creencias irracionales y asertividad}

$\mathrm{Al}$ observar las matrices de correlaciones entre los ítems de la variable Creencias Irracionales y los ítems de la variable Asertividad, podemos describir lo siguiente:

\section{Creencia 1}

Se observan 7 correlaciones significativas mayores al 20\% (en valor absoluto) con los ítems de Asertividad.

\section{Creencia 2}

Podemos observar 5 correlaciones significativas mayores al 20\%; acá resaltan particularmente las correlaciones del ítem 1 (Asertividad) con los ítems 12 (0.201), $22(0.224)$ y $32(0.251)$ de creencias irracionales.

\section{Creencia 3}

Podemos observar que no hay ninguna correlación significativa mayor a $20 \%$, sin embargo destacan las correlaciones significativas del ítem 43 de creencias irracionales con los ítems $17(0.142), 18(0.151)$ y $19(0.136)$ de asertividad.

\section{Creencia 4}

Podemos observar que solo hay dos correlaciones significativa mayores a $20 \%$, destacan las correlaciones significativas del ítem 34 de creencias irracionales con los ítems 3 (0.239), 9 (-0.179), $10(0.160)$ y $11(0.185)$ de asertividad. También las correlaciones significativas del ítem 3 de asertividad con los ítems $34(0.239), 74(-0.241)$ y $84(-0.145)$ de creencias irracionales.

\section{Creencia 6}

Podemos observar que solo hay una correlación significativa mayor a $20 \%$, destacan las correlaciones significativas del ítem 86 de creencias irracionales con los ítems 1 (-0.155), 13 (0.156), $18(0.191)$ y 19 (0.237) de asertividad.

\section{Creencia 7}

Podemos observar 8 correlaciones significativas mayores al 20\%; acá resaltan particularmente las correlaciones del ítem 3 (Asertividad) con los ítems 7 (0.262) y 17 (0.240) de creencias irracionales. También destacan las correlaciones del ítem 77 de creencias irracionales con los ítems $12(0.268)$ y $16(0.238)$ de asertividad.

\section{Creencia 8}

Podemos observar 6 correlaciones significativas mayores al 20\%; acá resaltan particularmente las correlaciones del ítem 68 de creencias irracionales con los ítems 4 (0.245), 6 (0.208), $12(0.266)$ y $16(0.241)$ de asertividad.

\section{Creencia 9}

Podemos observar 7 correlaciones significativas mayores al 20\%; acá resaltan particularmente las correlaciones del ítem 19 de creencias irracionales con los 
ítems $3(0.262)$ y $11(0.211)$ de asertividad. También destacan las correlaciones significativas del ítem 10 de asertividad con los ítems $69(0.207)$ y $79(0.232)$ de creencias irracionales.

\section{Creencia 10}

Podemos observar 2 correlaciones significativas mayores al 20\%; acá resaltan particularmente las correlaciones del ítem 50 de creencias irracionales con los ítems $1(0.202)$ y $3(0.206)$ de Asertividad.

Por todo lo anterior, en cuanto a la validación de la hipótesis interpretamos que si bien existen correlaciones, estadísticamente muy precisadas, estas son muy bajas, por lo tanto afirmamos que se rechaza la $\mathrm{H}_{0}$ y se acepta la $\mathrm{H}_{1}$.

\section{CONCLUSIONES}

1. Creencias irracionales con actos de violencia escolar.- La variable creencias Irracionales con la variable de actos de violencia están correlacionadas parcialmente y las que más se correlacionan son los ítems 1, 2 y 3 . Esto significa que existe una ramificación de la primera variable para juzgar los actos de violencia de los escolares, sin embargo se articulan con las tres primeras situaciones de actos de violencia, positivamente la primera y tercera e inversamente la segunda, referida a los criterios de una actuación drástica hacia el juicio de los actos de violencia de los estudiantes. En otras palabras, no existe un criterio racional amplio que se esperaría de un docente para abordar el tema de violencia, sin que ello signifique permisividad.

2. Asertividad con actos de violencia.- El ítem 1 de conductas violentas es el que más se correlaciona con los ítems de asertividad. También acá cabe resaltar que las correlaciones significativas estadísticamente hablando son bajas (la mayor en valor absoluto es de 0.279 o $27.9 \%$ ).

3. Creencias irracionales con asertividad.- Entre la creencia irracional 7 y los ítems de la variable asertividad se encuentran las mayores correlaciones significativas. Del mismo modo es pertinente aclarar que si bien es cierto existen correlaciones significativas estadísticamente hablando, las correlaciones siguen siendo bajas (la mayor en valor absoluto es de 0.268 o $26.8 \%$ ).

\section{REFERENCIAS BIBLIOGRÁFICAS}

Anales de Salud Mental (2009). Confiabilidad y validez de los cuestionarios de los estudios epidemiológicos de salud mental de Lima y de la selva peruana. Lima: ASM, Órgano oficial del Instituto Especializado en Salud Mental Honorio Delgado - Hydeyo Noguchi.

Arbulú, Ricardo (2002). Efectos de un entrenamiento en lenguaje positivo en un grupo de padres de familia. Lima: UNMSM.

Barron, Margarita (2006). Volencia Córdova: Brujas. 
CNE (2005). Proyecto Educativo Nacional. Propuesta del Consejo Nacional de Educación Lima: CNE.

CNE (2010). Propuestas de políticas de educación del Consejo Nacional de Educación. Educación para la sostenibilidad del crecimiento económico y la mejora de la calidad de vida de los peruanos. Lima: CNE.

Delors, Jacques (2000). Informe de la UNESCO de la Comisión Internacional sobre la educación para el siglo XXI. En Revista El futurista. Lima: Instituto de Estudios del futuro de la UNMSM.

Ellis, Albert (1990). Razón y emoción en la psicoterapia. Bilbao: DESCLEE.

Encinas Garza, José Lorenzo (1994). Bandas juveniles. Perspectivas teóricas. México: Trillas.

Fernández, Isabel (2005). Escuela sin violencia: Resolución de conflictos Lima: Alfaomega.

Hoyos, Olga, Aparicio, José, Córdova, Paola (2005). Caracterización del maltrato entre iguales en una muestra de colegios en Barranquilla (Colombia). En Revista Psicología desde el Caribe. Colombia: Universidad del Norte.

Lafarga, Juan y Gómez, José (1978). Desarrollo del potencial humano. Aportes de una psicología humanista. México: Trillas.

Martìnez Migueles, Miguel (1982). La psicología humanista. Fundamentación epistemológica, estructura y método. México: Trillas.

Ministerio de Educación (2002). Magisterio, educación y sociedad en el Perú. Una encuesta sobre opiniones y actitudes. Lima.

Ministerio de Salud del Perú (2001). Plan Nacional para la atención integral de salud del escolar y adolescente 2001-2006. Lima: MSP.

Monclús, Antonio y Saaban, Carmen (2006). La violencia escolar. Actuaciones y propuestas a nivel internacional. Barcelona: Davinci.

Municipalidad de Lima Metropolitana (2012). Proyecto educativo metropolitano, Lima ciudad educadora, documento base Lima, ciudad para todos.

Orellana M. Oswaldo (2005). Psicología y meztizaje, una aproximación histórica y psicoeducativa. En Revista de Investigación en Psicología, Vol. 8 N. ${ }^{\circ} 1$ Lima: IIPSI UNMSM.

Orellana M. Oswaldo y Orellana G. Daphne (2010). Estudio sociométrico de escolares que participanen actos violentos callejeros. En Memorias del III Congreso Iberoamericano y VII Congreso Nacional de Profesionales y estudiantes de Psicología. Lima: UIGV.

Rodríguez, Ernesto y equipo GTZ (2006). Políticas públicas y marcos legales para la prevención de la violencia relacionada con adolescentes y jóvenes. Estado del arte en América Latina 1995-2004 Lima: OPS GTZ.

Tong, Federico y Aragón, Rolando (2006). Políticas públicas, legislación y prevención de la violencia en jóvenes Lima: OPS GTZ. 
CREENCIAS IRRACIONALES Y ASERTIVIDAD DOCENTE CON ACTOS DE VIOLENCIA EN ESCOLARES

\section{ANEXO 1 \\ RELATOS DE CONDUCTA DOCENTE FRENTE A SUS ESTUDIANTES}

INSTRUCCIONES: Colega lee atentamente los tres relatos y responde a las preguntas solicitadas.

\section{PRIMER RELATO}

Jacinto es un Profesor Tutor de un salón de clase y ha detectado que un grupo de alumnos, que representa el $25 \%$ del aula, participa en pandillas callejeras. Frente a este hecho, el profesor Jacinto, acude a la delegación policial para denunciar a cada uno de ellos, logrando que la policía intervenga y los deposite a una celda, librándose así transitoriamente de esta anomalía social.

\section{SEGUNDO RELATO}

Dos colegas del profesor Rolando, lo emplazan para que haga un parte de disciplina, para expulsar de la institución educativa a un grupo de alumnos indisciplinados. El profesor Rolando, no atiende dicha petición y como alternativa y en su condición de tutor, elabora un programa de convivencia escolar y compañerismo, y en sesenta días, logra superar el desajuste disciplinario demostrando a sus colegas que es posible modificar situaciones relativamente difíciles en la formación escolar.

\section{TERCER RELATO}

Claudio es un profesor que cumple al día su programación pedagógica y es apreciado por colegas, alumnos y padres de familia. Un día un alumno se acerca a él para pedirle que intervenga frente a la situación de ser víctima de un compañero del aula, que le pide dinero para no maltratarlo, con la amenaza permanente de desprestigiarlo frente a otros compañeros como cobarde y homosexual. El profesor Claudio le manifiesta al alumno-víctima que no puede hacer nada porque esa no es su función.

\section{PREGUNTAS Y RESPUESTA}

1. ¿Es Correcta la conducta del profesor Jacinto? Primer relato SÍ NO

2. ¿Es Correcta la conducta del profesor Rolando? Segundo relato Sí NO

3. ¿Es Correcta la conducta del profesor Claudio? Tercer relato SÍ NO

4. ¿Alguna vez afrontaste una situación similar al primer relato? SÍ NO

5. ¿Alguna vez afrontaste una situación similar al segundo relato? SÍ NO

6. ¿Alguna vez afrontaste una situación similar al tercer relato? Sí NO 